\title{
Factores subjetivos y objetivos incidentes en el rendimiento académico en la carrera Banca y Finanzas, FAREM-Carazo, UNAN-Managua
}

MSc. José Daniel Guido Chávez

Docente investigador UNAN-MANAGUA, FAREM-CARAZO josielguivez@hotmail.com

Palabras clave: Rendimiento Académico, Factores subjetivos, Factores objetivos

\section{RESUMEN}

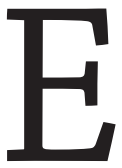

1 "Rendimiento Académico Estudiantil" es uno de los desafíos más grandes que enfrenta toda institución educativa superior para determinar la calidad de enseñanza que administra dentro del sistema educativo y uno de los mecanismos utilizados para medirla. Otra de las interrogantes que se perfila en el sistema educativo es ¿la evaluación del rendimiento académico de los estudiantes que realiza la institución educativa superior está considerando cada uno de los factores que se asocian a él? El conjunto de factores subjetivos y objetivos que tienen incidencia en este parámetro y la amplitud de su aplicación hacen que su valoración sea compleja. Mediante la aplicación de las técnicas de recolección de datos cualitativos y cuantitativos - encuestas, entrevistas, grupo focal a estudiantes - y ser procesados según la naturaleza de cada variable sujeta a estudio, se identifican los factores con mayor incidencia en el rendimiento académico.

\section{INTRODUCCIÓN}

El rendimiento académico de los estudiantes, es una función relativa de factores subjetivos como características sociodemográficas, motivacionales, vocacionales, socioeconómicos, gestión de las asignaturas del eje de investigación, pedagogía y metodología de 
enseñanza, formas de culminación de estudios, entre otros; y factores objetivos, refiriéndose a la procedencia de los estudios secundarios, retención estudiantil, deserción estudiantil, promoción estudiantil, características socio-productivas del estudiante, rigor científico de las asignaturas del eje de investigación, eficiencia terminal de los estudiantes (Lamas, 2008; Nuñez, 2009; UNAN-Managua, 2011).

Este término es muy conocido y debatido en toda la comunidad educativa desde muchas perspectivas por los grupos quela integran, desdelos estudiantes, los docentes, los representantes del sistema educativo, hasta la sociedad en general. Este término es muy complejo en todas las dimensiones que abarca su concepto en todo el sistema educativo de una nación. Navarro (2003) cita que, uno de los conceptos que se ha adoptado para referirse al rendimiento académico son las calificaciones que el estudiante obtiene en su práctica académica:

“[...] el indicador del nivel educativo adquirido, ha sido, sigue y probablemente seguirán siendo las calificaciones escolares. A su vez, estas son reflejo de las evaluaciones y exámenes donde el alumno ha de demostrar sus conocimientos sobre las distintas áreas o materias, que el sistema considera necesarias y suficientes para su desarrollo como miembro activo de la sociedad" (Cascón, 2000: 1-11).

Para efectos teóricos se describen algunas concepciones de investigadores que han estudiado los factores que inciden en el rendimiento académico y que además, son retomados en el estudio.

Lamas (2008) expresa que, la relación que tiene la motivación con el aprendizaje:

[...] parece haber coincidencia entre los distintos autores en vincular a la motivación intrínseca con aquellas acciones realizadas por el interés que genera la propia actividad, considerada como un fin en sí misma y no como un medio para alcanzar otras metas. En cambio, la orientación motivacional extrínseca, se caracteriza generalmente como aquella que lleva al individuo a realizar una determinada acción para satisfacer otros motivos que no están relacionados con la actividad en sí misma, sino más bien con la consecución de otras metas que en el campo escolar suelen fijarse en obtener buenas notas, lograr reconocimiento por parte de los demás, evitar el fracaso, ganar recompensas, etc. (p. 16)

Negrete (2009, citado por Argentina, 2013) expresa que, alguien que tenga un buen hábito de estudio significa saber cómo administrar el tiempo, mejorar la concentración y la memoria, leer libros de texto, tomar apuntes, escribir temas e informes, presentar exámenes, hacer informes orales, mejorar la motivación escolar y las relaciones interpersonales (p. 10).

Hay que resaltar, que para llegar a formar estos hábitos es evidente que el ejercicio sea todos los días, considerando una hora uniforme. En la formación de hábitos es necesario el ejercicio; pero, la práctica no conduce al aprendizaje. Saber si lo que hace está bien o mal, incita al individuo a modificar su conducta para que sea más eficiente en su estudio. Para llegar a formar 
los hábitos de estudio es necesario contar con tres factores principales como son psicológicos, ambientales e instrumentales.

Ruiz (2001) expone, que un estudiante procedente de un entorno familiar carencial tiene más posibilidades de obtener un bajo rendimiento en sus estudios puesto que demuestra capacidades intelectuales inferiores, presentando ritmos de aprendizajes y niveles de concentración lentos. Otra variable que está ligada es la formación que tienen los padres, no solamente por pertenecer a un determinado nivel social por los ingresos que obtiene, sino por los estímulos hacia el estudio, por las actitudes hacia el trabajo escolar y por las expectativas futuras depositadas en él (p. 89).

Las metodologías didácticas no son estáticas, sino que han evolucionado en conjunto con la evolución que tiene el hombre en la sociedad, es decir, que para dar soluciones al comportamiento socio-académico del ser humano se han implementado nuevas metodologías didácticas, así como mejoras didácticas a las que ya están establecidas. Para Garbanzo (2007a), las metodologías de estudio son "condiciones cognitivas" definiéndolas como "estrategias de aprendizajes que el estudiante lleva a cabo relacionadas con la selección, organización y elaboración de los diferentes aprendizajes" (p. 50).

Hablar de metodología didáctica, además de métodos, es también tratar con estrategias didácticas. En el Modelo Educativo Normativa y Metodología para la Planificación Curricular de la Universidad Nacional Autónoma de Nicaragua, Managua (UNAN-Managua) se encuentran algunas estrategias didácticas que ayudan a los docentes en su práctica metodológica, bajo el paradigma de que ellas están centradas en el estudiante: Ubicación Contextual, Guías de cuestionamiento de lo que se aprende, Observación auto-reflexiva, Aprendizaje colaborativo, Estudios de caso, Aprendizaje por proyectos, Aprendizaje basado en la resolución de problemas, e Informe escrito analítico-reflexivo, Trabajo de campo, Conferencias magistrales (pp. 38-40).

Debido a la situación socioeconómica que están presente en la sociedad, padres e hijos creen que estudiar y trabajar no son actividades compatibles, o que si un universitario busca un trabajo de medio tiempo es probable que baje sus notas; creen que es una carga demasiado grande para la época universitaria (Silva, 2011). Planas y Enciso (2014) en su estudio plantea, que las diversas razones por las cuales se trabaja mientras se estudia una carrera universitaria son:

Por necesidad económica, para ganar autonomía personal mediante la remuneración económica, para adquirir experiencia/currículum, los que estudian porque trabajan, o sea, ya trabajaban antes de estudiar, para estudiar mejor, o porque la carrera ya les proporciona suficiente experiencia (p. 28). 


\section{MATERIALES Y MÉTODOS}

El método de investigación del presente estudio es observacional, según el propósito del diseño metodológico el tipo de estudio es descriptivo (Piura, 2006). De acuerdo al tiempo de ocurrencia de los hechos y registro de la información, el estudio es retrospectivo y según el período y secuencia del estudio es longitudinal. De acuerdo al alcance de los resultados el estudio es analítico (Canales, Alvarado y Pineda, 1996). En cuanto al enfoque filosófico, por el uso de los instrumentos de recolección de la información, análisis y vinculación de datos, la presente investigación promueve la integración sistémica de métodos y técnicas cualitativas y cuantitativas, por tanto, se implementa un enfoque mixto (Pedroza 2016).

A partir de los datos que fueron recolectados mediante la aplicación de una encuesta a todos los estudiantes del quinto año de la carrera de Banca y Finanzas activos dentro de la cohorte comprendida 2011-2015, equivalente a 39 estudiantes, se diseñó la base datos correspondiente, utilizando el software estadístico SPSS, v. 20 para Windows, además, se aplicó entrevistas y grupo focal a los sujetos de estudio.

\section{RESULTADOS}

De los datos obtenidos de la aplicación de encuesta, entrevista y grupo focal a los estudiantes se definen los siguientes resultados:

Dentro de los factores motivacionales identificados en los estudiantes se tienen que el $72 \%$ de ellos eligió la carrera de Banca y Finanzas porque les gustaba y en una cuantía menor, pero significativa, el 23\% tenía conocimientos previos sobre la preparación intelectual en la carrera. Además, se muestra que el 79\% de los estudiantes optaron por la profesión para adquirir habilidades y destrezas en el campo; así mismo, el 67\%, en obtener conocimiento científico en la ciencia de las finanzas, también en la facilidad de inserción al campo laboral; y el 62\%, por el auge que tiene este sector en nuestra economía. Un elemento que se suma a la motivación de los estudiantes es que el $97 \%$ conocía el pénsum de la carrera, es decir, tenían conocimiento pleno del proceso académico en el que estarían y el desempeño profesional que tendrían en el futuro (figuras 1, 2 y 3). Esta información estadística se complementa con lo expresado por estudiantes entrevistados quienes mencionan, que la decisión de estudiar la carrera de Banca y Finanzas es por sentirse atraído por el mundo de las finanzas, el mover del dinero en la economía, realizar análisis e interpretación financiera de proyectos, rentabilidad de una inversión, búsqueda de soluciones financieras tanto a personas jurídicas como naturales, entre otras actividades financieras y la habilidad de manejar escenarios vinculados con operaciones cuantitativas. 


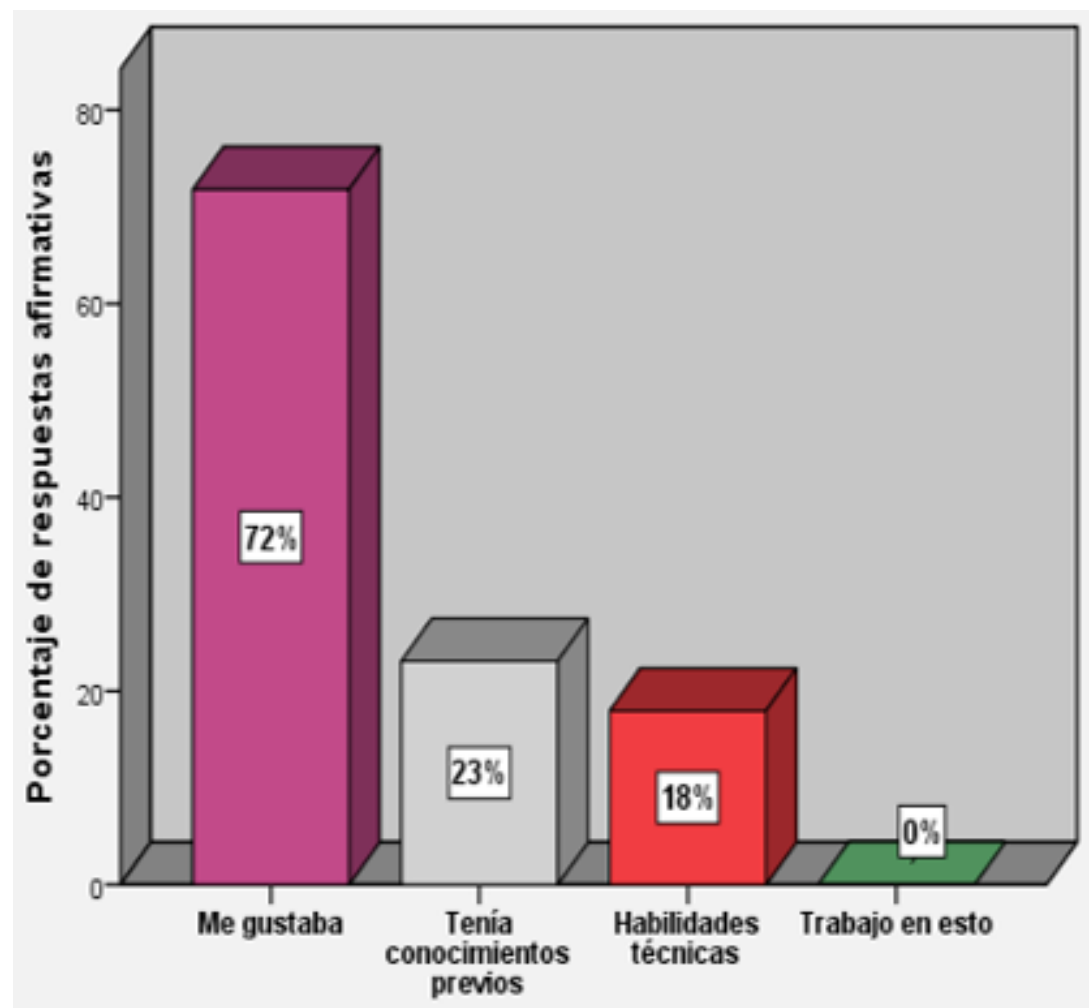

Figura 1. Motivación personal

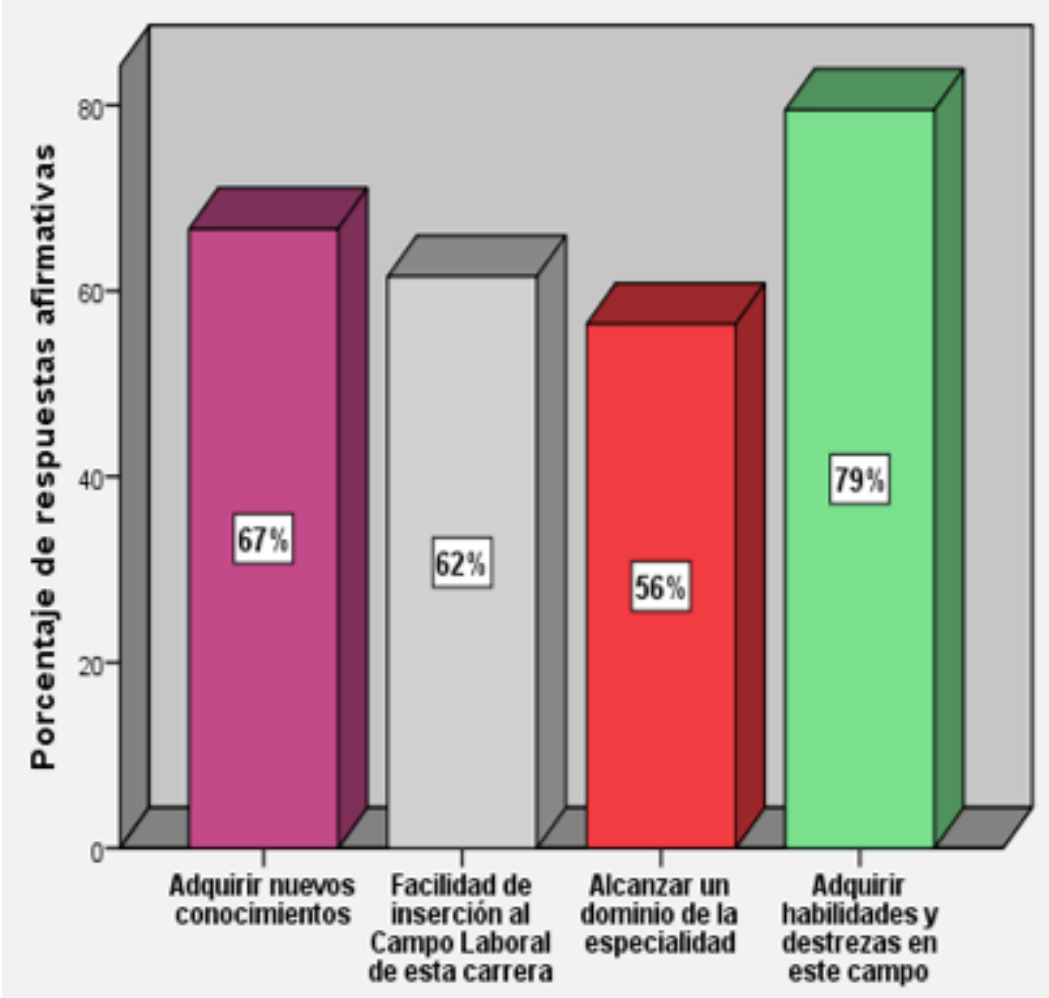

Figura 2. Qué esperas de tu carrera 


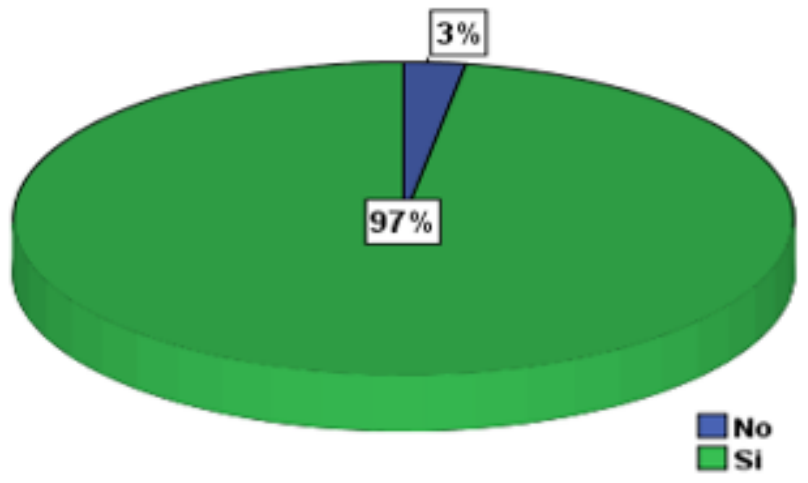

Figura 3. Conoce el Pensum de su carrera

Otro elemento que se valoró, es la responsabilidad académica de los estudiantes, referidas a la frecuencia de asistencia a las sesiones de clase, obteniendo un 97\% en la categoría siempre, lo que indica que los estudiantes están comprometidos a alcanzar el objetivo que se plantearon al ingresar sus estudios universitarios; un $66 \%$ de puntualidad, revelando un comportamiento regular de cumplimiento en su ejercicio académico y en un 95\% manifiestan la conciencia en que, entregar las tareas en tiempo y forma, permite obtener resultados académicos de aprobación en sus asignaturas (figuras 4,5 y 6 ).

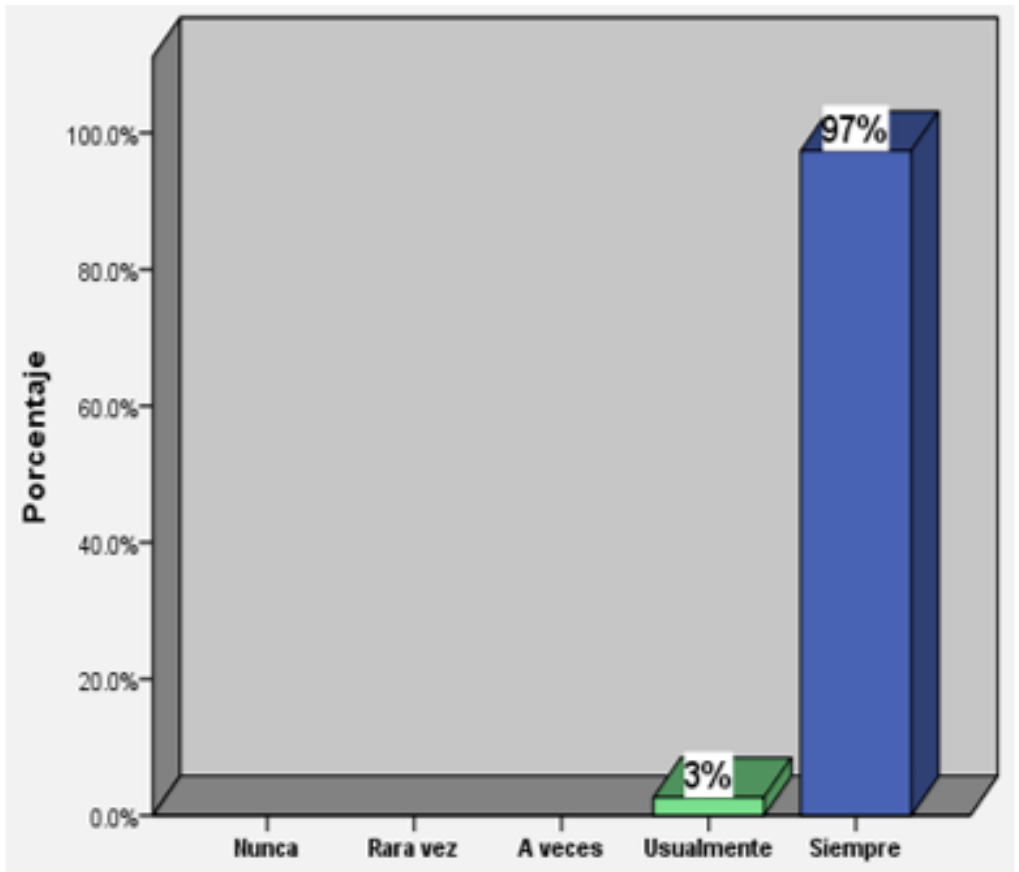

Figura 4. Frecuencia de asistencia 

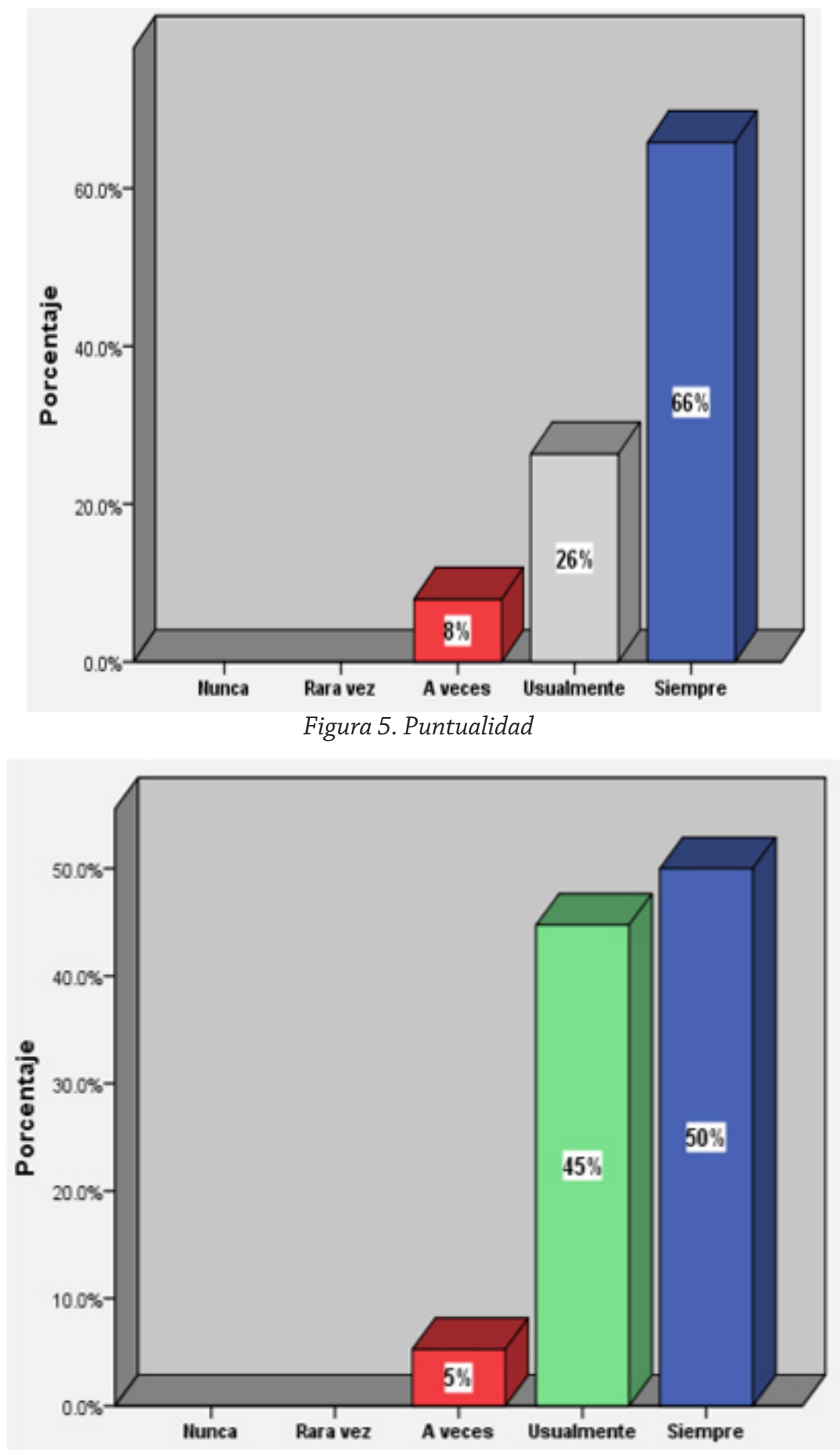

Figura 6. Entrega de tareas en tiempo y forma

No se puede descartar aquellos componentes que tuvieron un impacto negativo en la motivación de las personas como lo representan los factores exógenos: en el campo académico -como lo plantea la teoría- el docente influye sobremanera en el estudiante mediante la metodología y pedagogía usada en el proceso enseñanza-aprendizaje, que según los datos del estudio refleja un $36 \%$ de impacto en ellos (figura 7). 


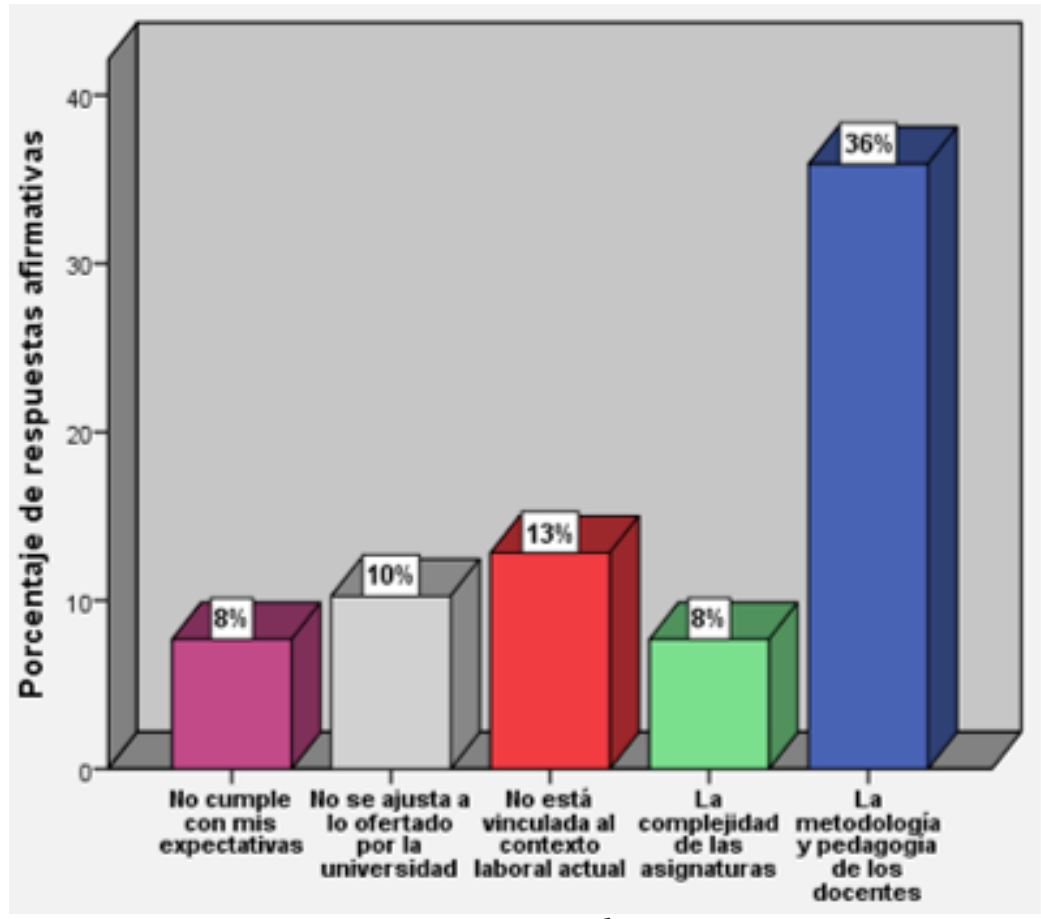

Figura 7. Factor que desmotiva

Al describir que los estudiantes presentan una buena responsabilidad académica, hay que destacar la frecuencia de estudio particular que poseen, al igual que los métodos con los que disponen para su preparación independiente: respecto a la frecuencia de estudio, se observa que el $51 \%$ lo realizan usualmente; el $26 \%$ lo hace en la categoría a veces y tan solo el $15 \%$ en categoría siempre. El tiempo que los estudiantes dedican para el estudio independiente muestra un promedio de 2.90 horas diarias.

Se determina, que el método de estudio independiente que realizan los estudiantes consiste en un $87 \%$ en el uso de Internet, seguido del $51 \%$ en consulta a un compañero que tiene alto dominio de las asignaturas y en tercer lugar, con $49 \%$ visitas a la biblioteca (figuras 8,9 y 10). 


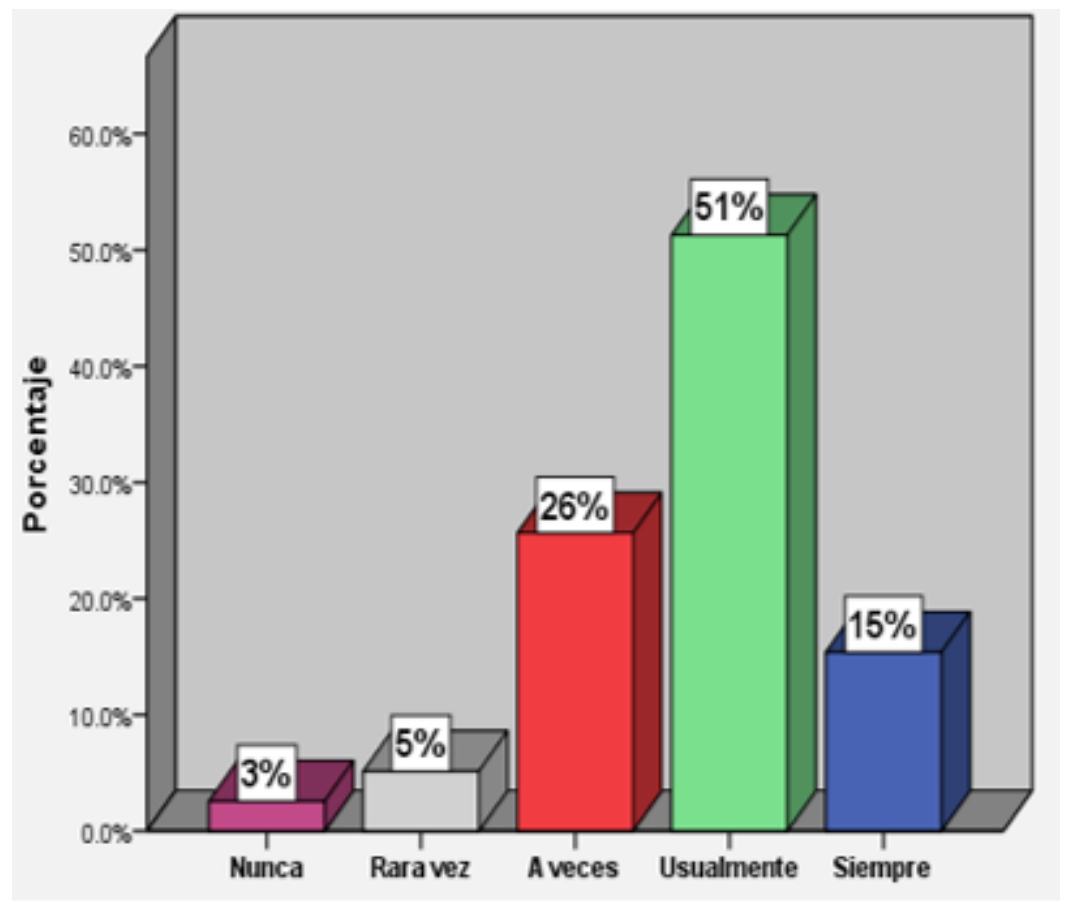

Figura 8. Frecuencia de estudio independiente

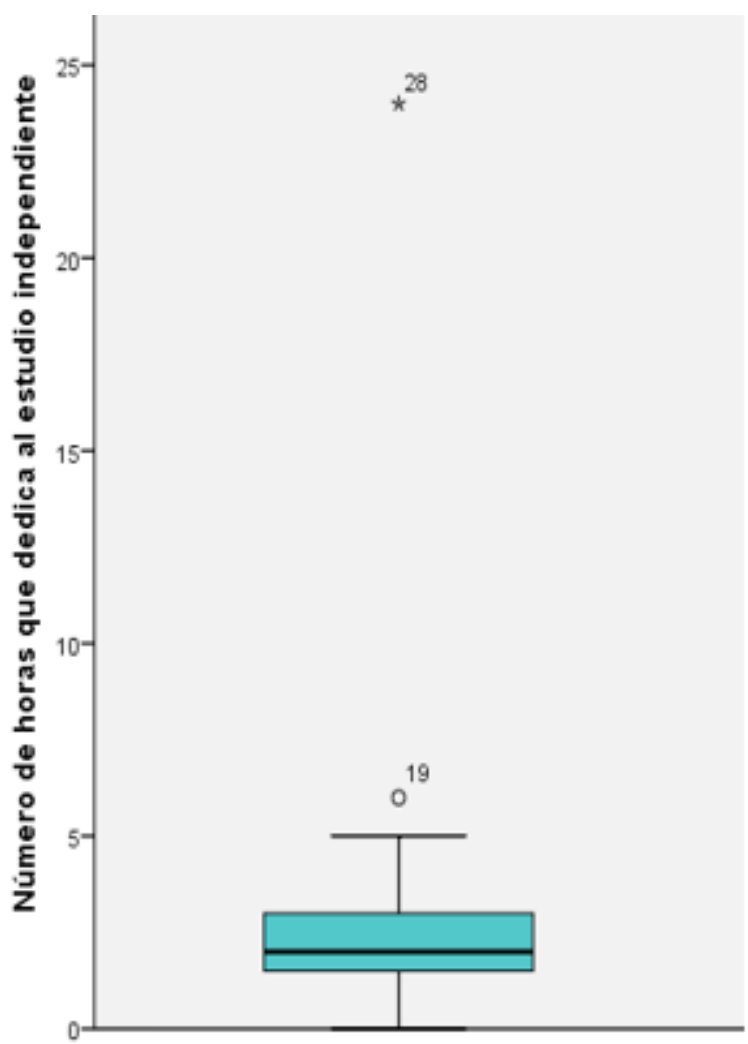

Figura 9. Horas de estudio 


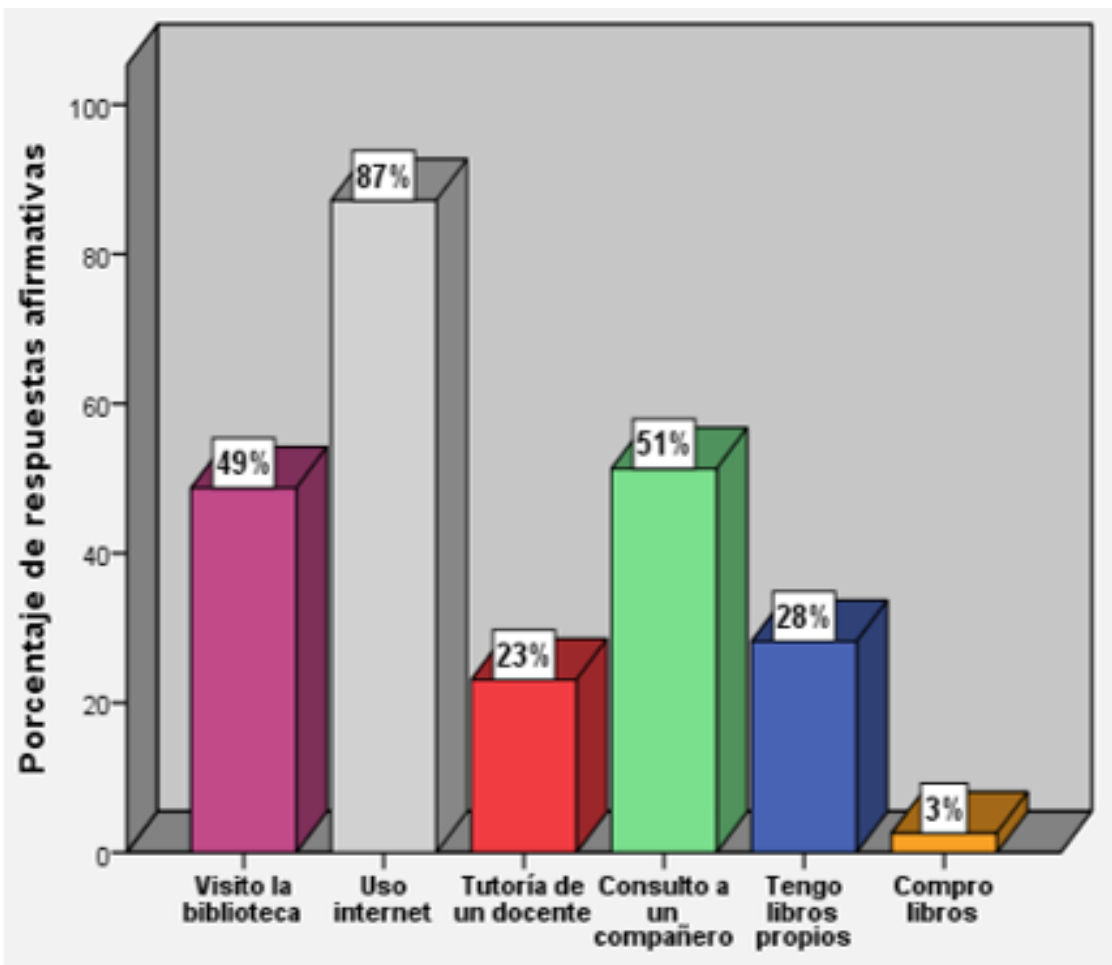

Figura 10. Método de estudio independiente

Respecto al uso de Internet, los datos reflejan que el $64 \%$ de los estudiantes utilizan los cibercafé como uno de los lugares de acceso a la búsqueda de información y el $51 \%$ accede en la universidad. Todo lo anteriormente descrito tiene una frecuencia del $63 \%$ de forma usual.

Cuando se refirió al método consulta a un compañero, el resultado final muestra que lo realizan en una forma usual, y esto se soporta con el hecho de que constituye el segundo método de estudio utilizado. En el método de estudio visita a la biblioteca se puede destacar que los estudiantes tienen un promedio de 1.4 horas diarias en que el $70 \%$ de ellos lo realizan con un parámetro entre 1 a 4 horas (figuras 11 y 12 ). 


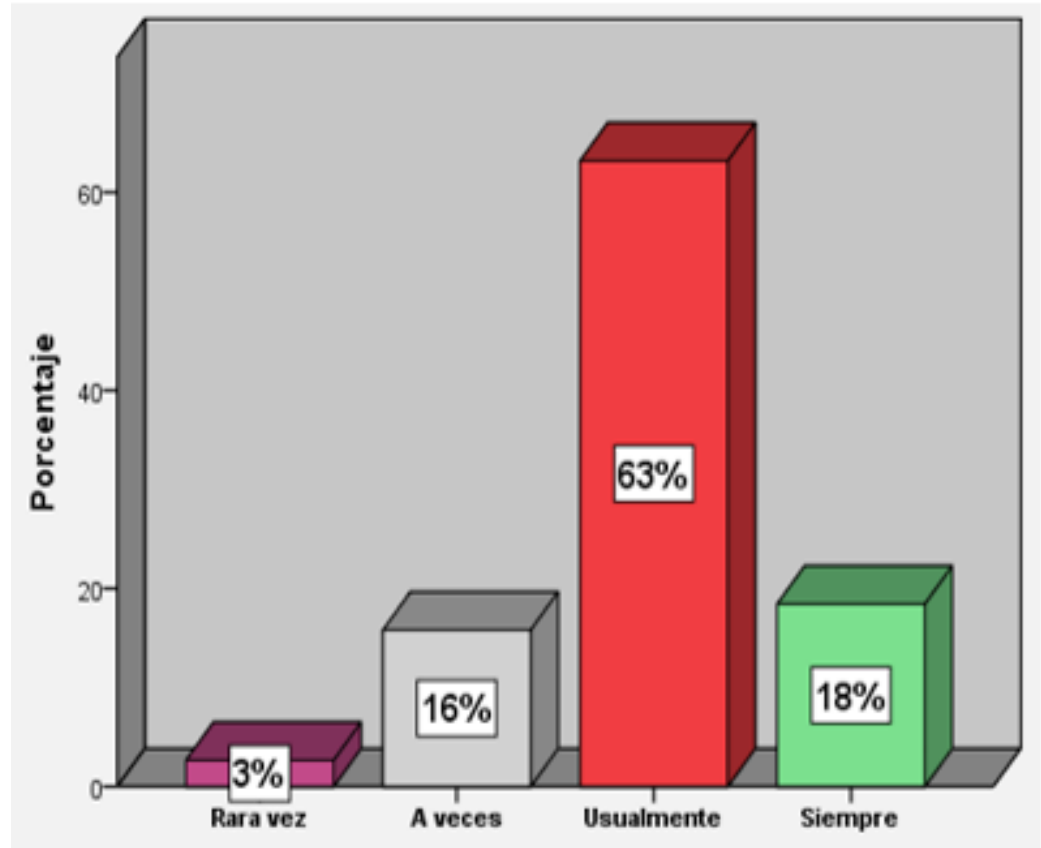

Figura 11. Frecuencia de uso de Internet

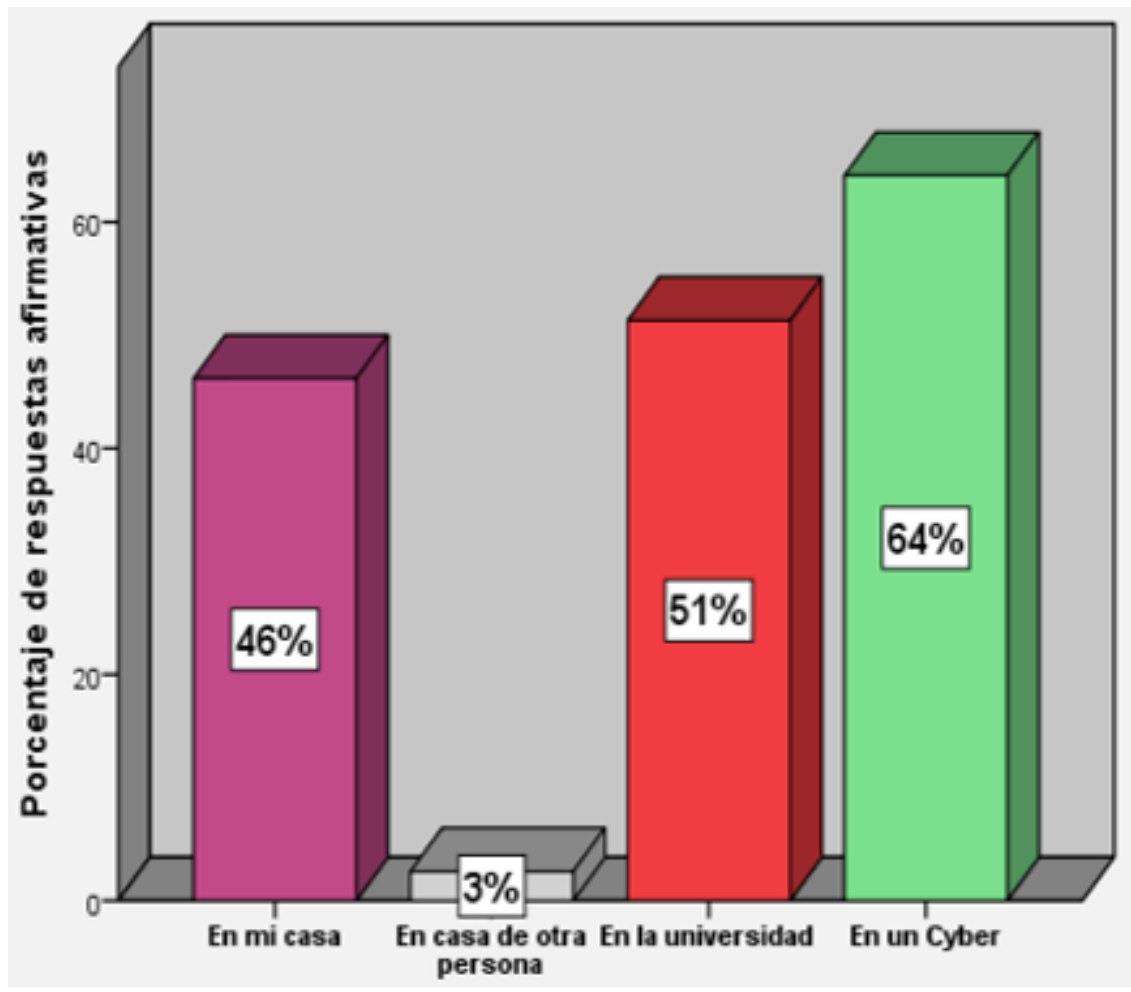

Figura 12. Lugar de acceso a Internet

Un aspecto que valorar es que, estos métodos de estudio independiente no ocurren de forma individual, sino que los estudiantes realizan mezcla de ellos y de esta forma alcanzan el objetivo de tener una preparación mejor en cada una de las asignaturas que cursan en los semestres. 
Contrastando los datos estadísticos descritos anteriormente conla información cualitativa obtenida de las entrevistas, se comprobó, que a lo largo de la vida académica universitaria los estudiantes, han presentado hábitos de estudio buenos, lo que ha permitido mantener resultados cuantitativos en la categoría muy buena, moviéndose en promedios generales entre 80 - 89 (datos provenientes de los registros académicos del grupo en estudio).

Partiendo de las referencias cualitativas extraídas referente a la forma de enseñanza de los docentes que impartieron la clase y el cómo ha influido esto en su desempeño académico, en primer lugar, se describió de forma general, que en su mayoría, quienes han impartido clase en el grupo, se han identificado en ser docentes que todavía practican enfoques con métodos tradicionalistas, quienes utilizan el dictado, ponencias con materiales bastante teórico y poco práctico, abusos en proporcionar folletos en los que el estudiante se dedica a la lectura de dicho material, profesores no comprometidos con la labor docente, evidenciado en la poca preparación didáctica en las sesiones de clase, así como conversatorios de temas de la disciplina no acordes al contenido a abordarse, docentes que desmotivan al grupo por no hacer uso de metodologías activas, poco uso de las tecnologías de la información y la comunicación (TIC).

Hay que destacar, que existen docentes con buenas metodologías de enseñanza, señalándoles como excelentes, que han ayudado a cambiar ese paradigma tradicional de los demás, pero si se pone en una balanza, se cuenta con más docentes tradicionales que los que han podido formarles académicamente bien (palabras tomada de un participante del grupo focal y avalado por el resto). Los estudiantes consideran, que estas actuaciones docentes son factores determinantes en el desempeño académico que les permite alcanzar resultados académicos cuantitativos y cualitativos de calidad o resultados mediocres.

En términos generales, se abordó la forma de evaluación de sus docentes a lo largo de los cinco años de la carrera. Las opiniones extraídas de los instrumentos de recolección cualitativos revelaron que también sobresale lo tradicional, es decir, utilizaron mecanismos de valoración de los saberes como pruebas sistemáticas, exposiciones, trabajos grupales, investigaciones documentales. Un elemento a resaltar es que los docentes, en su mayoría, cumplen las orientaciones establecidas por los órganos rectores encargados de orientar la modalidad de evaluación de los aprendizajes.

No se puede pasar por alto, aquellos casos en los que las evaluaciones fueron realizadas con menor rigor científico, que no contribuyeron a mejorar el aprendizaje mediante retroalimentaciones, simplemente se realizaban con la finalidad de hacerlas. Las declaraciones de los entrevistados revelaron escenarios en donde los docentes no le dieron el valor pertinente a la evaluación, se olvidaron del objetivo primordial que llevan consigo evaluar. Ellos divulgaron situaciones, como es el caso de los trabajos investigativos y las exposiciones con calificación 
estándar, pero, no se valoró guiándose por una rúbrica de evaluación sino que se hizo de forma superficial. Las pruebas escritas fueron aplicadas con interrogantes cuyas respuestas debían ser contestadas teóricamente iguales, otros las elaboraron extensas, comparando contenido demandado con el tiempo asignado para realizarlas y se agrega otro componente negativo, como es la baja puntuación.

Los estudiantes expresaron, además, situaciones en la que los contenidos de las asignaturas no se abordaron con calidad metodológica, pedagógica y científica, añadiendo la desvinculación entre lo enseñado con lo demandado por las empresas del sector financiero, temáticas inconclusas, porque el docente no tuvo dominio científico completo de la materia que impartía, no se hizo uso de software que permitiera vincular el conocimiento teórico impartido con lo ejecutado en los sistemas empresariales, puesto que la facultad no cuenta con laboratorios contables-financieros (infraestructura, paquetes tecnológicos) básicos, en donde se pudiera practicar los distintos escenarios que ocurren en las empresas y que a su vez, agilizara y dinamizara el proceso de aprendizaje. Hay discontinuidad de enfoques metodológicos, pedagógicos, didácticos y de conocimiento cuando se da la ocurrencia de cambios de docentes por circunstancias inesperadas. Todo esto tiene un impacto grande en la calidad del aprendizaje y en el desempeño futuro como profesional de los estudiantes.

No todo es negativo, se tiene presencia de educadores comprometidos con su labor docente, cuya finalidad es lograr obtener una educación de calidad. Los estudiantes encuestados mencionaron, que en diversos casos, los docentes incluyeron tópicos que no estaban en el programa de asignatura pero que eran complementarios para ampliar los saberes de esa asignatura. También se presentó la intención de un docente en enseñar temas específicos, pero por no contar con las condiciones apropiadas (laboratorios de computación no disponibles) para realizar el ejercicio, entonces fracasó la idea.

Se ha descrito en forma general, cómo ha sido la vivencia académica vista desde la perspectiva estudiantil, en la que la ocurrencia de factores endógenos y exógenos inciden en los resultados académicos de manera favorable o desfavorable, dependiendo de la situación sociocultural-emocional que posea el estudiante. Es importante señalar las perspectivas que tienen los docentes en el desarrollo del proceso educativo desde el comportamiento del estudiante hasta su aportación en la enseñanza, la metodología utilizada, la forma de evaluación, entre otros aspectos:

Los estudiantes de la cohorte 2011-2015, presentaron evolución en su comportamiento académico, en el que algunos eran despreocupados en sus primeros años cuando se hacían en grupos de trabajos, por ejemplo, al dividirse los grupos, integrando a tres participantes únicamente, los que mantuvieron esa actitud negativa se motivaron a ser cumplidores (se vieron 
obligados). Algunos que estudian diplomados, paralelamente con los estudios de grado, se les ayudó a complementar el conocimiento, se convirtieron en estudiantes investigativos que no se quedaron con la información del docente.

El comportamiento del estudiante es una variable dependiente del comportamiento del docente, que es influenciado por la capacidad, destreza, conocimiento que tenga el docente en su materia. Cuando hay dominio, se tiene motivación, interés, preocupación en el estudiante por superar esa asignatura, porque, si no se prepara se reprueba la clase. Por el contrario, si no hay dominio del docente, el estudiante se desmotiva, se sale de clases, no estudia, se fresquea, no asiste. Se observa que los estudiantes le dedican mayor atención a las clases con mayor rigor (clases con temáticas numéricas, en la que la práctica de los saberes se mantiene en todo el desarrollo del semestre) que aquellas que son bastante teóricas.

La filosofía tiene que ver con la formación del docente. Si este ha sido preparado para ser educador, posee una filosofía orientada a la docencia con herramientas de enseñanzas dirigidas a mejorar la calidad de los aprendizajes, esto se manifestará en el futuro al trasmitir sus conocimientos en el quehacer diario. En cambio, el que llega a la universidad sin tener preparación docente, simplemente se dedicará a trabajar por el salario y no por enseñar; para tener un cambio, transcurrirá un buen tiempo para comprender la filosofía docente. La formación puede ser desde estudios normalistas hasta estudios superiores como diplomados, maestrías, otros. Se debe destacar, que este escenario de enseñar trasciende las fronteras de las instituciones educativas y se manifiesta también en las empresas en donde la necesidad de transmitir el saber a otro compañero es inevitable no cumplirlo, porque es un proceso de evolución dentro de ella.

En la FAREM-Carazo, el cuerpo docente aplica, en el desempeño académico, estrategias didácticas descritas en el modelo educativo de UNAN-Managua, en las diversas asignaturas que posee el pénsum de la carrera, de tal forma que ayuden a ubicar a los estudiantes en los distintos contextos en los que van a estar inmersos en el campo laboral. Se han aplicado estrategias como la ubicación contextual sumando con técnicas como debates, discusiones, la parte analítica de los escenarios que ayudará a construir el conocimiento. Toda empresa enfrenta problemas y tiene que dar solución a los mismos. Con los estudios de casos, nuestros estudiantes aprenden a dar soluciones a esos problemas, además, aprenden a trabajar de forma colaborativa, que es un elemento indispensable en toda empresa.

La evaluación está estrechamente ligada a las estrategias que se utilizan y el área científica de la asignatura. Otro aspecto, es la capacidad que tiene el docente en equilibrar la forma de evaluación para incidir en el rendimiento académico del estudiante; ella no es rígida y se debe adaptar a la personalidad del grupo. 
También existen algunos elementos que tienen una influencia negativa y que se pudo detectar en el grupo como son:

- El cambio de la conformación de grupos impacta en los estudiantes por los esquemas que tienen.

- La participación pasiva en el transcurso de la clase, mostrando un comportamiento callado.

- Apatía al trabajar en grupo cuando no se unen por afinidad, se presentaba confrontaciones en las personalidades de los miembros del grupo.

- Resistencia al cambio en todos sus ámbitos. Como ejemplo, la dependencia que tienen por la edad de ellos.

- El uso de la tecnología de una forma inadecuada, como es el caso del apego de los celulares que en clase están siendo usados.

- Motivación (desinterés) de terminar un proyecto que han iniciado.

- Problemas familiares, enfermedades de los estudiantes, comunicación entre ellos.

\section{DISCUSIÓN DE RESULTADOS}

Tras la revisión teórica y el posterior estudio empírico, es de importancia resaltar las aportaciones fundamentales que deja marcado la ejecución de la investigación en el área académica referida al rendimiento académico. Se presentará tomando en consideración las variables que se plantearon en los objetivos.

Los hogares de los estudiantes en estudio, enfrentan uno de los retos más grande que puede afrontar una familia en la sociedad nicaragüense como lo es la situación socioeconómica. La característica común de estos hogares es que, tomando la variable ingresos económicos, se encuentran en un estatus social medio, puesto que en casi la mitad de los casos logran obtener entradas de dinero que les permite cubrir la mitad del costo del que actualmente cuesta la canasta básica en la nación, situación que desfavorece en gran cuantía la obtención de todos los recursos que necesita tener un estudiante, el alcance de una buena preparación académica. Sin duda alguna, en vista de la situación, los estudiantes toman decisiones de incursionar al mundo laboral, aun estando estudiando, como apoyo a sus familias, para costear los gastos que incurren en la preparación profesional en la educación superior.

En los resultados empíricos, se observa que, los estudiantes que presentan esta situación, representan tan solo el 29\%, es decir, que el restante depende de sus padres, situación que demuestra que a pesar de no disponer de todos los recursos, gran parte de ellos se dedican 
solamente a la actividad educativa. Se añade, además, una variable que justifica a que los estudiantes no estén con necesidad de buscar empleo de forma inmediata, dicha variable es no tener hijos a su cargo ni personas que estén bajo su obligación, en su mayoría, indicando que disponen de mayor espacio para el estudio. Este escenario es un factor favorable, debido a que el mayor esfuerzo del estudiante está centrado en alcanzar resultados cuantitativos altos, resultados que, según registros académicos, muestran que su efecto es positivo en los estudiantes sujetos de estudio, tal a como lo refleja el indicador de promoción, que tiene una tendencia creciente respecto a promoción limpia.

La existencia de variables (interna como externa al individuo) se reflejaron bajo la terminología de factores subjetivos y objetivos, tratando de abarcar componentes personales como también institucionales. Una de las variables que tiene influencia significativa es la motivación que tiene el estudiante en realizar las actividades ligadas a los estudios. La literatura presenta sinnúmero de manifestaciones que involucra la labor de emprender una acción o dejar de realizarla por la presencia de factores que alteran el estado de ánimo, complementándose con la otra variable vocación para realizar la actividad con interés. El estudio se dirigió en conocer cuáles fueron los determinantes para que los estudiantes estudiaran la carrera de Banca y Finanzas tomando estos parámetros:

En la fase empírica del estudio, se reflejó claramente, que la elección de estudiar la carrera fue movida por la preferencia (gusto) del estudiante hacia las actividades financieras, al igual que identificarse con la parte numérica de la ciencia. Los datos empíricos mostraron que casi los tres cuartos (72 \%) del estudiantado les gustó la carrera por motivos de conocimiento sobre las temáticas que se iban a abordar en el tiempo de su preparación académica, al igual, aquellas aportadas por las interacciones sociales como la familia (apoyo a su hogar, alcanzar un mejor estatus económico y por consiguiente, social y otros), indirectamente las amistades, la necesidad de independizarse, la divulgación (publicidad) por la carrera referida al campo laboral en donde se desempeñaría, entre otros. La demostración de este hecho está reflejada en las cifras expresadas por el indicador eficiencia terminal que mantuvo índices porcentuales excelentes por encima de $95 \%$, reafirmado con la excelente tasa de retención durante la cohorte (moviéndose entre 96\% $-100 \%)$.

Un elemento influyente en el desempeño logrado por el estudiantado producto de la motivación desde sus diversos ámbitos, fueron los hábitos de estudio que mantuvieron durante el periodo académico. Los datos estadísticos recolectados en la investigación respondieron a las diversas publicaciones teóricas relacionadas a las metodologías que adoptaron los estudiantes en el proceso de aprendizaje. La frecuencia de estudio de forma individual y grupal demostró ser buena, es decir, la responsabilidad de estudio se cumplió por arriba del 50\% según la categoría de usualmente y siempre. Además, se afirma que el estudiantado hizo buen uso del tiempo 
destinado a su preparación personal fuera de la interacción con el docente ya que los resultados arrojan que mantuvo un promedio de 2.90 horas dedicada a estudiar, acompañada con distintos recursos o formas de estudio.

Las formas de estudio independiente, mayormente usadas por el estudiantado fueron tres: consultas a sitios Web (uso de Internet con el 87\%), consultas a un compañero de clase (51\%) que tenía bastante dominio de las asignaturas y consultas en libros de texto (visita a la biblioteca con el 49\%). Se afirma, que todo ello tuvo efecto positivo en el desempeño de los estudiantes, logrando alcanzar el rendimiento académico cuantitativo por arriba de los 80 puntos lo cual lo califica en un resultado muy bueno.

Un dato desalentador en la motivación del estudiantado es la utilización de enfoques tradicionalistas en la metodología y la pedagogía de los docentes en el desarrollo de su enseñanza, los estudiantes tienen conocimiento del impacto que produce este tipo de actitud en su aprendizaje. La investigación refleja, desde las opiniones estudiantiles, la controversia existente entre las diversas teorías orientadas en dinamizar la enseñanza mediante la aplicación de estrategias y técnicas que promuevan la adquisición de saberes significativos, pertinentes y relevantes con las prácticas de los docentes. Estos están conscientes de las exigencias que trae consigo el entorno educativo, los retos que constantemente enfrentan ante las demandas de la sociedad en ofrecer de una educación con calidad y los desafíos en tener una preparación profesional con conocimientos contextualizados ligados con los avances tecnológicos. Se conoció, que la universidad invierte en capacitaciones periódicas para mantener actualizados y preparados a sus docentes sobre los avances metodológicos a nivel internacional. A pesar de las acciones de mejoras al desempeño del docente, se mantiene la resistencia al cambio en adoptar nuevos enfoques educativos.

\section{CONCLUSIÓN}

Con base a los resultados obtenidos en la investigación se establece que los factores subjetivos y objetivos identificados con mayor incidencia en el rendimiento académico son: motivación personal, vocación hacia la carrera, hábitos de estudios, ingreso familiar, procedencia, influencia de las TIC en la metodología y pedagogía docente y metodologías en el proceso enseñanza-aprendizaje

\section{LISTA DE REFERENCIAS}

Argentina, A. (2013). "Relación entre hábitos de estudio y rendimiento académico en estudiantes de primero básico" (estudio realizado en el instituto básico de educación por cooperativa, San Francisco la Unión,
Quetzaltenango). Universidad Rafael Landívar, Quetzaltenango.

Canales, Alvarado y Pineda. (1996). Metodología de la Investigación, Manual 
para el Desarrollo de personal de Salud. Oficina Regional de la Organización Mundial de la Salud, OPS (2da ed.). Washington, D.C. 20037, E.U.A.

Garbanzo, G. (2007a). Factores asociados al rendimiento académico en estudiantes universitarios, una reflexión desde la calidad de la educación superior pública. Revista Educación, 31(1), 43-63.

Lamas, H. (2008). Aprendizaje autorregulado, motivación y rendimiento académico. Liberabit, 14(14), 15-20.

Núñez, J. (2009). Motivación, aprendizaje $y$ rendimiento académico. Actas do $\mathrm{X}$ Congresso Internacional GalegoPortuguês de Psicopedagogia., 41-67.

Pedroza, M. (2016). Uso de los Modelos Univariados, Regresión y Correlación, en estudios de Tipo Observacional. Managua: UNAN - Managua.

Piura, J. (2006). Metodología de la Investigación Científica: Un Enfoque Integrador (1ra ed.). Managua: PAVSA.
Planas, J. y Enciso, I. (2014). Los estudiantes que trabajan: ¿tiene valor profesional el trabajo durante los estudios? Revista iberoamericana de educación superior, 5(12), 23-45.

Ruíz, C. (2001). Factores familiares vinculados al bajo rendimiento. Revista Complutense de Educación, 12(1), 81-113.

Silva., M. (2011). “Universitarios que estudian y trabajan: una realidad que debemos atender" Universidad César Vallejo. Recuperado 02 de junio 2016, http:// maestrosendocenciauniversitaria5. webnode.es/news/universitarios-queestudian-y-trabajan-una-realidad-quedebemos-atender/

UNAN - Managua. (2011). Modelo Educativo, Normativa y Metodología para la Planificación Curricular 2011. Managua: UNAN - Managua. 